\title{
Control of Gluconeogenic Growth by pps and pck in Escherichia coli
}

\author{
YUN-PENG CHAO, ' RANJAN PATNAIK, ' WILLIAM D. ROOF, ${ }^{1,2}$ RYLAND F. YOUNG, ${ }^{2}$ \\ AND JAMES C. LIAO ${ }^{1,2 *}$ \\ Department of Chemical Engineering ${ }^{1}$ and Department of Biochemistry and Biophysics, ${ }^{2}$ \\ Texas A\&M University, College Station, Texas 77843
}

Received 24 May 1993/Accepted 31 August 1993

\begin{abstract}
It is well-known that Escherichia coli grows more slowly on gluconeogenic carbon sources than on glucose. This phenomenon has been attributed to either energy or monomer limitation. To investigate this problem further, we varied the expression levels of pck, encoding phosphoenolpyruvate carboxykinase (Pck), and pps, encoding phosphoenolpyruvate synthase $(\mathrm{Pps})$. We found that the growth rates of $E$. coli in minimal medium supplemented with succinate and with pyruvate are limited by the levels of Pck and Pps, respectively. Optimal overexpression of $p c k$ or $p p s$ increases the unrestricted growth rates on succinate and on pyruvate, respectively, to the same level attained by the wild-type growth rate on glycerol. Since Pps is needed to supply precursors for biosyntheses, we conclude that $E$. coli growing on pyruvate is limited by monomer supply. However, because pck is required both for biosyntheses and catabolism for cells growing on succinate, it is possible that growth on succinate is limited by both monomer and energy supplies. The growth yield with respect to oxygen remains approximately constant, even though the overproduction of these enzymes enhances gluconeogenic growth. It appears that the constant yield for oxygen is characteristic of efficient growth on a particular substrate and that the yield is already optimal for wild-type strains. Further increases in either Pck or Pps above the optimal levels become growth inhibitory, and the growth yield for oxygen is reduced, indicating less efficient growth.
\end{abstract}

When a bacterium grows in a batch culture without any nutrient limitation or product inhibition, the growth rate in the exponential phase, defined as the unrestricted growth rate or balanced growth rate, depends on the carbon source used. For Escherichia coli in minimal medium, the order is $k_{\text {acetate }}<$ $k_{\text {succinate }}<k_{\text {pyruvate }}<k_{\text {glycerol }}<k_{\text {glucose }}$ (1), where $k$ is the specific growth rate with the carbon source indicated. This unrestricted growth rate is an inherent metabolic characteristic of the bacterium, and its control differs from the growth rate control in chemostat cultures, where cells are constantly limited by a nutrient.

For batch cultures, Anderson and von Meyenburg (1) found that the oxygen consumption per minute per cell remains approximately the same, whereas the unrestricted growth rates differ significantly with various carbon sources. For chemostat cultures, Hempfling and Mainzer (16) found that the maintenance energy, i.e., the ATP produced extrapolated to the zero dilution rate, is higher when the cell growth is limited by a poor carbon source such as acetate. Therefore, the low growth rates on poor carbon sources have been commonly attributed to the high energy requirement for the biosynthesis of key metabolites (17). Recently, Marr (20) argued that growth on poor carbon sources is limited by metabolite precursor supply rather than by energy demand. He showed that the metabolic flux distribution at the branch points for anabolic and catabolic reactions may be suboptimal when cell growth is supported by poor carbon sources.

Bacterial growth rate is usually varied by shifting nutritional conditions in batch cultures or by controlling the dilution rate in chemostat cultures. To investigate further the growth rate control on poor carbon sources, we varied the expression levels of two gluconeogenic enzymes, phosphoenolpyruvate carboxykinase (Pck) (encoded by $p c k$ ) and phosphoenolpyruvate

\footnotetext{
* Corresponding author.
}

synthase (Pps) (encoded by pps), whose enzymatic activities are required for growth on succinate or pyruvate. Pps converts pyruvate to phosphoenolpyruvate (PEP) when cells are growing on minimal medium containing pyruvate (Fig. 1). Pps competes with pyruvate dehydrogenase (Pdh) which mediates the formation of acetyl coenzyme A, the starting metabolite of the tricarboxylic acid cycle. When the cells grow on succinate, Pck converts oxaloacetate to PEP, which then bifurcates to gluconeogenesis and to the formation of pyruvate. Pck has to compete with citrate synthase (GltA) for a common substrate, oxaloacetate. Mutants with null alleles of pps cannot grow on pyruvate (7). Both pps and pck null alleles are needed to generate the Suc phenotype (inability to grow on succinate), presumably because malic enzyme and Pps can supply PEP in $p c k$ cells (13). To control the Pck or Pps expression level, a pps $p c k$ strain was used and the $p c k^{+}$or $p p s^{+}$gene was expressed from a multicopy plasmid under the control of an inducible promoter. Unrestricted growth rates and specific oxygen consumption rates with either succinate or pyruvate as the sole carbon source were measured at different gene expression levels. Strains expressing inactive but stable Pps or Pck were used as controls.

\section{MATERIALS AND METHODS}

Materials. The following reagents were purchased from the indicated suppliers: D-glucose, amino acids, nucleotides, and all salts, Sigma Chemical Co. (St. Louis, Mo.); protein assay reagents and chemicals for sodium dodecyl sulfate-polyacrylamide gel electrophoresis (SDS-PAGE), Bio-Rad (Richmond, Calif.); isopropyl- $\beta$-D-thiogalactopyranoside (IPTG), USB (Cleveland, Ohio); restriction enzymes, Taq DNA polymerase, and T4 DNA ligase, Promega (Madison, Wis.); and the protein molecular weight standard, GIBCO BRL (Gaithersburg, Md.).

Bacterial strains and plasmids. The $E$. coli strains used are listed in Table 1. HG4 pps pck was a gift from Hugh A. Goldie, 


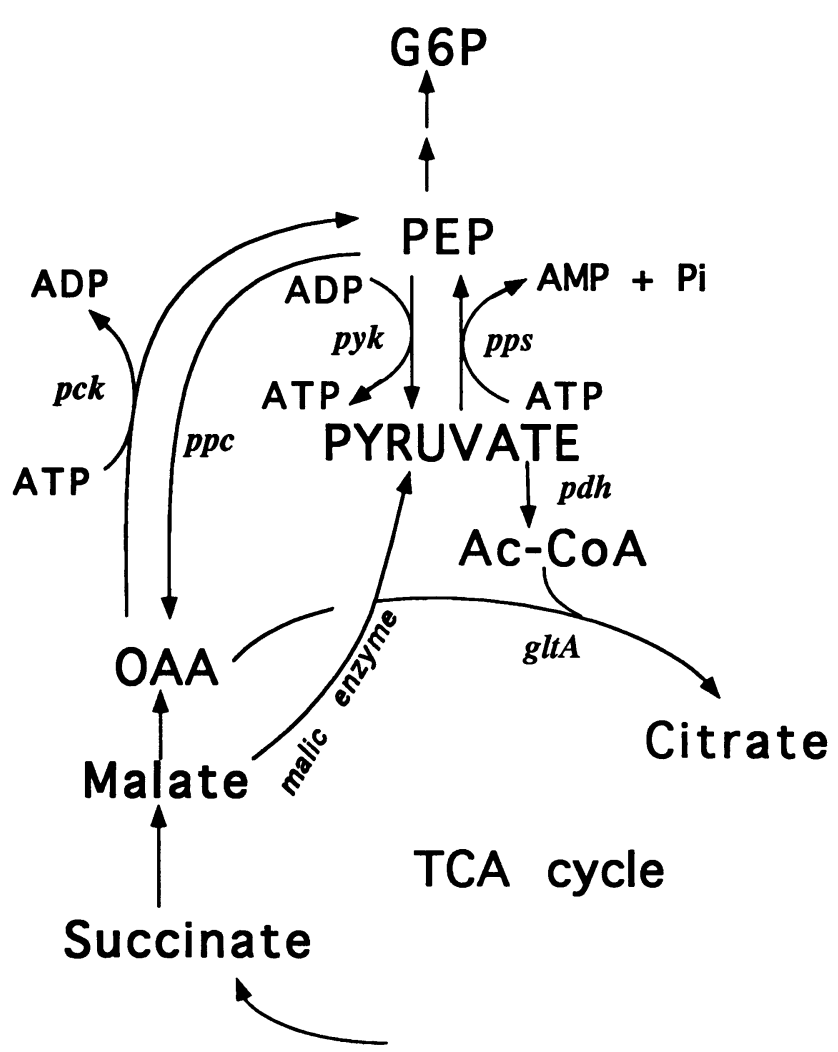

FIG. 1. Schematic of metabolic pathways in E. coli relevant to this study. Gene symbols: $p p s$, encoding PEP synthase; $p y k$, encoding pyruvate kinase; $p c k$, encoding PEP carboxykinase; $p p c$, encoding PEP carboxylase; $p d h$, encoding pyruvate dehydrogenase; glt $A$, encoding citrate synthase. Abbreviations: G6P, glucose 6-phosphate; Ac-CoA, acetyl coenzyme A; OAA, oxaloacetate; TCA, tricarboxylic acid.

University of Saskatchewan, Saskatoon, Saskatchewan, Canada. To construct $p p s^{+} p c k$ or $p p s p c k^{+}$strains, a P1 lysate from a pps $^{+}$pck $^{+}$strain (CGSC4456) was used to transduce HG4 pps pck to $\mathrm{Pyr}^{+}$(growth on pyruvate) or $\mathrm{Suc}^{+}$. Two transductants (one $\mathrm{Pyr}^{+}$and one $\mathrm{Suc}^{+}$) were picked and made $\mathrm{lacI}^{\mathrm{q} 1}$ and $\operatorname{rec} A$ by transducing to $\mathrm{Km}^{\mathrm{r}}$ and $\mathrm{Tc}^{\mathrm{r}}$ with $\mathrm{P} 1$ lysates from RY1912 and GE642, respectively.

Media and growth conditions. For strain construction, the cells were grown on Luria-Bertani (LB) medium (22). For physiological characterizations, all strains were cultured on M9 minimal medium (22) supplemented with a carbon source and the required nutrients as needed to overcome the auxotrophy. Cultures of HG4-derived strains were grown overnight on M9 with $0.2 \%$ glucose supplemented with tyrosine $(0.1 \mathrm{mg} / \mathrm{ml})$, histidine $(0.1 \mathrm{mg} / \mathrm{ml})$, and uracil $(0.02 \mathrm{mg} / \mathrm{ml})$. The cells were then washed twice in M9 medium without a carbon source and inoculated (initial cell density, ca. $10^{7}$ cells per $\mathrm{ml}$ ) in the fresh medium with either $0.5 \%$ succinate or pyruvate but without glucose. When the cultures reached mid-exponential growth phase (ca. $6 \times 10^{7}$ cells per $\mathrm{ml}$ ), they were diluted by the same medium to a cell density of $2 \times 10^{7}$ cells per $\mathrm{ml}$ and were cultured for two to three more generations. This dilution was to avoid potential limitation of oxygen transfer, depletion of nutrient components, and accumulation of toxic products. The last few generations showed a reproducible, steady-state growth rate which was taken as the unrestricted growth rate. All cultures were grown at $37^{\circ} \mathrm{C}$ on a rotary shaker, and ampicillin $(50 \mu \mathrm{g} / \mathrm{ml})$ was added as a selection pressure for plasmid-bearing strains.

DNA manipulations. The E. coli pck and pps genes were cloned by use of mini-Mu replicon Mu d5005 as previously described $(15,24)$. The mini-Mu plasmids containing pck or pps genes were selected on the basis of the ability to complement pck or pps mutations on the chromosome. These genes were recovered from the mini-Mu plasmids and subcloned into expression vector pUHE23-2 to yield pCK322 and pPS341, respectively. To eliminate the natural promoter and other control sequences, the structural genes were cloned by use of polymerase chain reaction (PCR). The primers were designed on the basis of the published sequences $(21,23)$, and they contain EcoRI and Bam HI sites on the $5^{\prime}$ - and $3^{\prime}$-end primers, respectively. The resulting PCR product of the pck gene is rid of its natural promoter but contains a putative natural ribosome binding site. The PCR product of pps is also without the natural promoter but contains a $\mathrm{T} 7 \phi 10$ ribosome binding site provided from the primer. The 1.7-kb Eco RI-Bam $\mathrm{HI}$ fragment of $p c k$ PCR product was ligated into pUHE23-2 to generate

TABLE 1. E. coli strains and plasmids used

\begin{tabular}{|c|c|c|}
\hline Strain or plasmid & Relevant characteristics & Source or reference \\
\hline \multicolumn{3}{|l|}{ Strains } \\
\hline HG4 & pck-2 pps-3 galK pyrD edd-1 his tyrA rpsL thi tonA & 12 \\
\hline JCL1053 & Same as HG4 but $l a c I^{41} \operatorname{rec} A$ & This study \\
\hline JCL1126 & Same as JCL1053 but $p c k^{+}$ & This study \\
\hline JCL1128 & Same as JCL1053 but pps ${ }^{+}$ & This study \\
\hline RY2157 & mutD5 galU5 & 9 \\
\hline RY1912 & lacI ${ }^{91}$ lac $Z:: \operatorname{Tn} 5$ & Laboratory collection \\
\hline GE642 & $\Delta r e c A z f i: \operatorname{Tn} 10$ & G. Weinstock \\
\hline CGSC4456 & sucA gal-25 $\lambda^{-} \operatorname{trp} A 9767$ iclR7 IN(rrnD-rrnE) & B. Bachmann \\
\hline \multicolumn{3}{|l|}{ Plasmids } \\
\hline pUHE23-2 & $\mathrm{Ap}^{\mathrm{r}}$; IPTG-inducible $\mathrm{T} 7$ (A1) early promoter (with lac $O$ insertion) & H. Bujard \\
\hline pCK322 & Same as pUHE23-2 but $p c k^{+}$(from mini-Mu plasmid) & This study \\
\hline pCK401 & Same as pUHE23-2 but $p c k^{+}$(PCR product) & This study \\
\hline pCK401m1 & Same as pCK401 but $p c k-50$ & This study \\
\hline pPS341 & Same as pUHE23-2 but $p^{+} s^{+}$(from mini-Mu plasmid) & 24 \\
\hline pPS341X1 & Same as pPS341 but pps-50 (2-codon insertion) & 24 \\
\hline pPS503 & Same as pUH23-2 but $\mathrm{pps}^{+}$(PCR product) & This study \\
\hline pPS560 & Same as pPS503 but pps-50 & This study \\
\hline
\end{tabular}




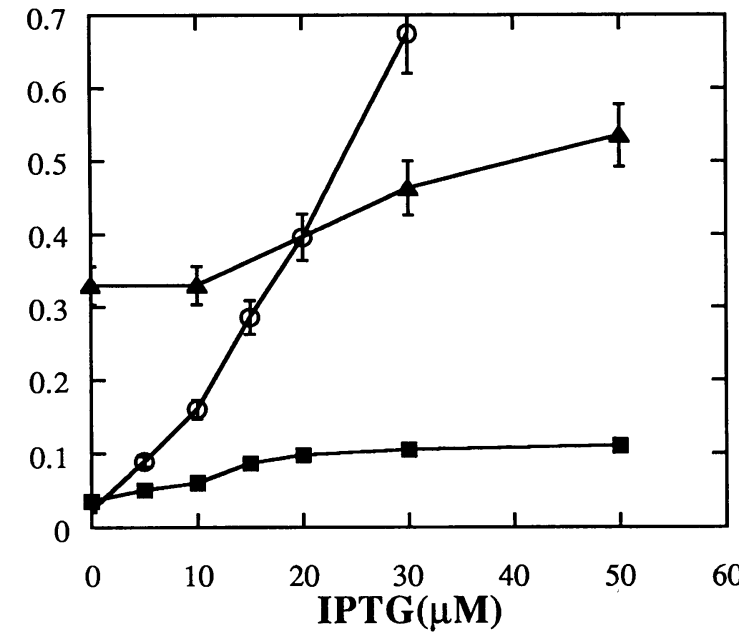

FIG. 2. IPTG-inducible expression of Pps and Pck activities. Symbols: $\bigcirc$, Pck activity in JCL1053 pps pck/pCK401 $p c k^{+} ; \boldsymbol{\Delta}$, Pps activity in JCL1053 pps pck/pPS341 pps $^{+} ; \mathbf{\square}$, Pps activity in JCL1053 pps $p c k /$ PPS503 pps $^{+}$.

pCK401. The PCR product of the pps gene, a 2.4-kb EcoRIBam HI fragment, was inserted into pUHE23-2 to yield pPS503. All DNA manipulations essentially follow the procedures published earlier (26).

Enzyme assays. Preparation of cell extracts was described elsewhere $(13,24)$. The Pck and Pps activities in these extracts were measured spectrophotometrically by monitoring the appearance of oxaloacetate and disappearance of pyruvate, respectively $(12,24)$. Total protein content was determined by use of the Bio-Rad dye reagent (Bradford assay) and bovine serum albumin as the protein standard.

Measurement of the oxygen consumption rate. A Clark-type polarographic oxygen probe was used to monitor the disappearance of oxygen from a sealed chamber. Experimental procedure was described previously (24).

Definition of variables. The specific growth rate $(k)$ is the fractional cell weight (dry weight) generated per hour, which is calculated as the inverse of doubling time multiplied by $\ln 2$. The specific oxygen consumption rate $\left(Q_{\mathrm{O} 2}\right)$ is the oxygen consumed per hour per unit (dry weight). The growth yield for oxygen $\left(Y_{\mathrm{O} 2}\right)$ is the dry weight of cells produced per unit of oxygen consumed, which in balanced growth is equal to $k / Q_{\mathrm{O} 2}$. The yield can be interpreted as the stoichiometry of growth with respect to oxygen in balanced growth. The flux through an enzyme is the rate of reaction mediated by the enzyme.

\section{RESULTS}

Overexpression of active and inactive Pck and Pps. Plasmids pCK401, pPS341, and pPS503 were transformed into a pps pck strain (JCL1053) and the IPTG-inducible activities of Pck and Pps were measured. As shown in Fig. 2, Pck activity can be varied over 20 -fold by IPTG with pCK401. However, pPS341 and pPS503 showed only two- to threefold induction in the presence of IPTG. It is not known why these two plasmids responded so little to the IPTG concentration. pPS341 always expressed higher Pps activities because of the presence of a natural pps promoter in the cloned fragment in the plasmid. SDS-PAGE shows that these plasmids yielded proteins with
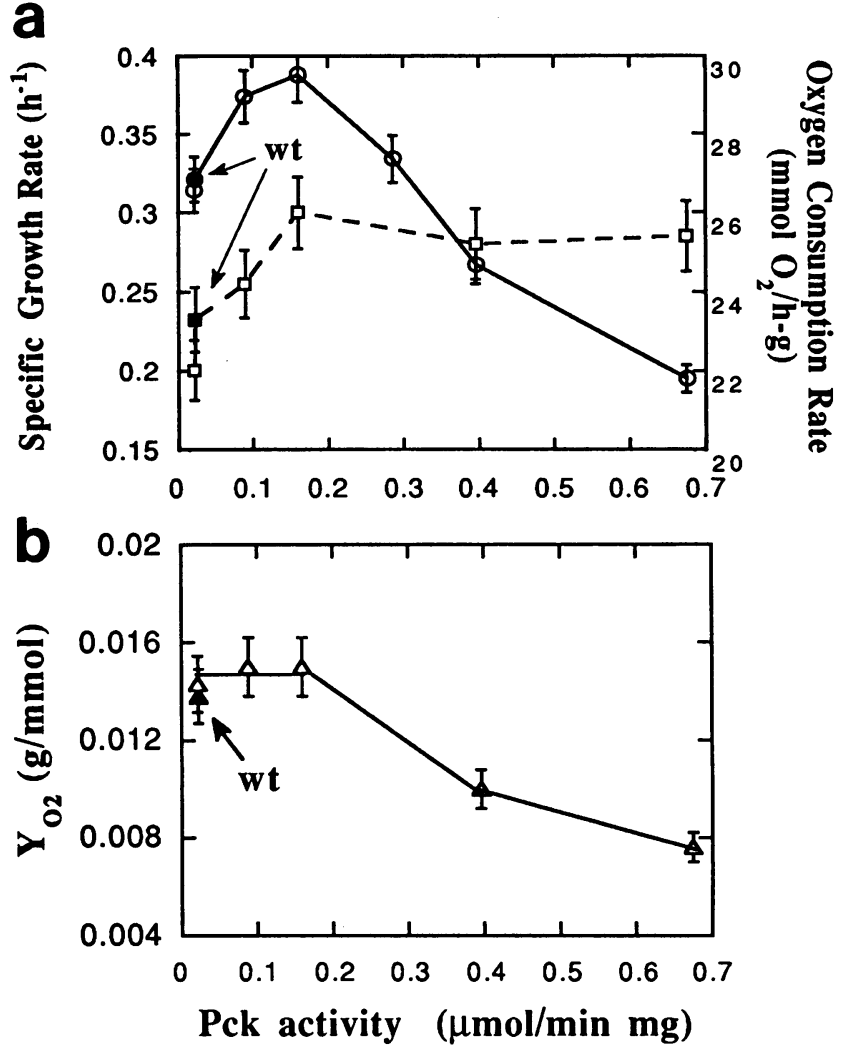

FIG. 3. Optimal Pck activity in E. coli growing on succinate is ca. 10 -fold higher than the wild-type level. (a) Specific growth rate $(O)$ and specific oxygen consumption rate ( $\square$ ); (b) growth yield for oxygen, $Y_{\mathrm{O} 2}=k / Q_{\mathrm{O} 2}$. These variables were measured from cultures of JCL1053 pps pck/pCK401 $p c k^{+}$at different Pck activities adjusted by IPTG concentration. The wild-type (wt) data were measured from cultures of JCL1126 pps $p c k^{+} / \mathrm{pCK} 401 \mathrm{~m} 1$, in which Pck activity was not dependent on IPTG.

the previously reported molecular weights (ca. 51,000 $M_{\mathrm{r}}$ for Pck and 80,000 $M_{\mathrm{r}}$ for Pps) $(11,21,23)$.

The inactivated Pck enzyme was isolated by transformation of pCK401 into a mutD5 strain (RY2157) (9). The plasmid DNA was then purified from the mutD5 strain and transformed into $\mathrm{HG} 4$ pps pck. The $\mathrm{Ap}^{\mathrm{r}}$ transformants were screened for Suc ${ }^{-}$but $\mathrm{Glc}^{+}$phenotype (growth on glucose). The plasmid from one such colony (designated pCK $401 \mathrm{~m} 1$ ) was purified and transformed into JCL1053 to confirm the phenotype. SDS-PAGE shows that pCK401m1 expressed approximately the same amount of Pck protein as pCK401 pck ${ }^{+}$ did, but no Pck activity was detected from the strain containing pCK $401 \mathrm{~m} 1$. It is therefore concluded that the mutation is in the $p c k$ structural gene. The mutant $p c k$ gene is then designated $p c k-50$. The inactivated Pps protein was constructed by a two-codon insertion, as described previously $(4,24)$. The inactivated Pps protein was stable and can be detected by SDS-PAGE with Coomassie brilliant blue staining (24).

Pck activity affects the growth rate and respiration on succinate. To assess the effect of Pck activity on the unrestricted growth rate on succinate, we used plasmid pCK401 to vary the Pck activity in a $p c k p p s$ strain. The unrestricted growth rate, Pck activity, and oxygen consumption rate were measured as described above at various IPTG concentrations. Figure $3 a$ shows the specific growth rate and the specific 
oxygen consumption rate of a JCL1053 pps pck/pCK401 pck ${ }^{+}$ in M9-succinate medium as a function of Pck enzyme activity. An isogenic strain (JCL1126/pCK401 m1) containing wild-type $p c k^{+}$on the chromosome and $p c k-50$ on the plasmid was used as a control under the same IPTG concentrations. The wildtype Pck activity expressed from the chromosome of this strain was suboptimal: an increase in Pck activity increased the unrestricted growth rate on succinate (Fig. 3a). However, the growth rate peaked and quickly decreased as the Pck expression level increased. The inactive Pck enzyme (expressed from pCK $401 \mathrm{~m} 1$ ) in the control strain did not affect the growth rate at low expression levels, indicating that the growth rate increase was Pck activity dependent. However, the inactive Pck enzyme also inhibited growth at high expression levels, but the effect is less significant than that caused by the active enzyme. The reason for the decrease in growth rate is unclear, although the ribosome burden caused by high-level protein production cannot be ruled out. No inclusion bodies were found in strains overproducing either active or inactive Pck.

As negative controls, JCL1053/pCK401 and an isogenic strain, JCL1128 $\mathrm{pss}^{+} / \mathrm{pCK} 401$, were grown on minimum medium supplemented with glucose or pyruvate. The growth rates on these two carbon sources were not affected by Pck activity at low expression levels (data not shown). Therefore, the increase in growth rate was specific to the carbon source. However, the growth rate decreased at high expression levels in minimal medium supplemented with pyruvate and with glucose, similar to that observed during growth on succinate.

In M9-succinate medium, the specific oxygen consumption rate of JCL1053 pps pck/pCK401 pck ${ }^{+}$(Fig. 3a) also increased as the Pck activity increased. However, the growth yield for oxygen $\left(Y_{\mathrm{O} 2}=k / Q_{\mathrm{O} 2}\right)$ remained approximately the same in this region (Fig. 3b). The oxygen consumption rate reached a plateau when the growth rate reached the maximum (Fig. 3a), indicating that the cell utilized oxygen (or energy) less efficiently when Pck level became inhibitory to growth. The yield coefficient decreased as a result.

Pps activity affects the growth rate and respiration on pyruvate. To probe the control of growth rate on pyruvate, we used pPS341 and pPS503 to vary the expression level of Pps. Unrestricted growth rate and oxygen consumption were measured as described above (Fig. 4a). A pps ${ }^{+}$strain (JCL1128) containing a plasmid-coded, inactivated Pps (from pPS341X1 or pPS560) was used as a control under the same growth conditions and inducer concentrations. Again, the wild-type Pps activity expressed from the chromosome was suboptimal for growth on pyruvate. The growth rate on pyruvate can be increased by moderately overexpressing Pps. However, excessive amounts of Pps inhibited growth. As a control, the growth rate was not dependent on the expression level of the inactivated Pps (coded by pps-50). Unlike overexpression of Pck, relatively high levels of inactivated Pps did not cause growth inhibition during growth on pyruvate. No inclusion bodies were detected in strains overproducing either active or inactive Pps.

Similar to the results in Fig. 3a, the oxygen consumption rate also increased with growth rate and remained constant when the growth rate decreased (Fig. 4a). The growth yield for oxygen (Fig. 4b) remained constant in the rising part of the growth rate curve and decreased when Pps activity increased beyond the optimal point. It is interesting to note that the maximum growth rates on succinate (Fig. 3a) and on pyruvate (Fig. 4a) are the same. In addition, this maximum rate is approximately the same as the unrestricted growth rate of this strain on glycerol $\left(0.40 \mathrm{~h}^{-1}\right)$.

Pps activity does not increase the growth rate on succinate. Pps activity can also support growth on succinate as long as the
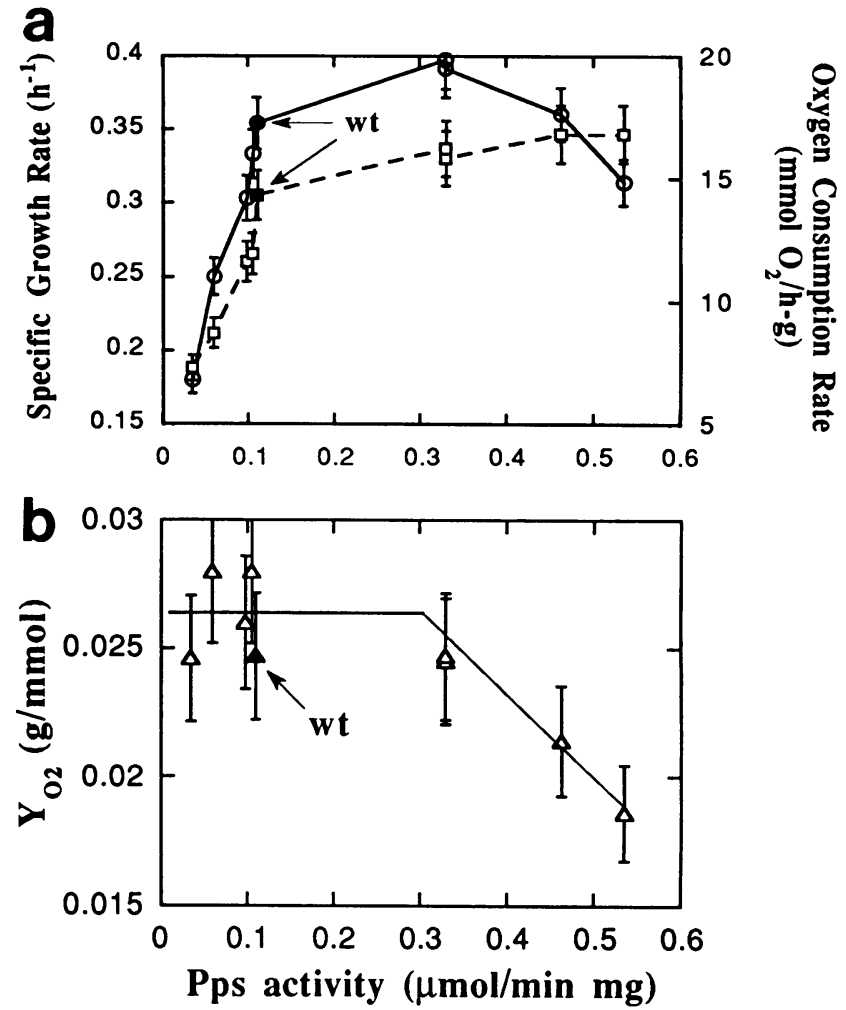

FIG. 4. Optimal Pps activity in E. coli growing on pyruvate is significantly higher than the wild-type level. (a) Specific growth rate (O) and specific oxygen consumption rate $(\square)$; (b) growth yield for oxygen, $Y_{\mathrm{O} 2}=k / Q_{\mathrm{O}}$. These variables were measured from cultures of JCL1053 pps pck/pPS503 pps ${ }^{+}$and JCL1053 pps pck/pPS341 pps ${ }^{+}$at different Pps activities adjusted by IPTG concentration. The wild-type (wt) data were measured from cultures of JCL1128 pps ${ }^{+} p c k / p P S 560$, in which Pps activity was not dependent on IPTG.

cell has malic enzyme, which converts malate to pyruvate. To assess the importance of Pps in growth on succinate, we varied the Pps expression level by use of pPS341 or pPS503 in a pps $p c k$ strain (JCL1053). It was found that the specific growth rate was $0.22 \mathrm{~h}^{-1}$, much lower than the $p c k^{+}$strain (Fig. 3). Moreover, the growth rate was not dependent on the IPTGinducible Pps activity (data not shown). Therefore, the conversion from succinate to pyruvate appears to be limiting in this case.

\section{DISCUSSION}

Although it is well-known that $E$. coli growth rates vary with the carbon source provided, the bases for this growth rate variation remain elusive. The most common explanation for the low growth rates in poor carbon sources is perhaps energy limitation. This view was supported by three lines of evidence: (i) the energy requirement for synthesizing the key metabolites (17) is higher on poor carbon sources than on glucose $(17,18)$; (ii) the respiration rates on poor carbon sources are roughly the same as that on glucose (1); and (iii) the maintenance energy is higher on poor carbon sources as measured in chemostat cultures (16). Although commonly cited, the first argument is a result of metabolic stoichiometry and should not be used alone in discussing the growth rate control. A cell with a low efficiency (an inefficient pathway) of energy production 
can still be limited in growth rate by fluxes to monomers, that is, by the availability of biosynthetic precursors.

Recently, Marr (20) argued that the low growth rates on poor carbon sources are caused by suboptimal fluxes leading to the key metabolites. This theory suggests that energy is not limiting and that the respiration rate is high relative to the growth rate because of suboptimal flux distributions. Marr's theory implies that it would be possible to increase the growth rate by increasing the flux to the limiting metabolites. This change would also improve the growth yield for oxygen, because less oxygen is wasted through the improved flux distribution. On the other hand, if the growth rate were limited by the energy production rate, one could increase the growth rate by increasing the flux to the energy-yielding pathways such as the tricarboxylic acid cycle.

We attempted to vary the flux distribution by varying the expression levels of Pps and Pck. We found that the effects of Pps and Pck overexpression can be divided into two parts. With low-level expression, overproduction of Pps or Pck enhances the growth rates on pyruvate and on succinate, respectively, up to a maximum (Fig. 3 and 4), which is higher than the wild-type growth rates on these carbon sources. However, the growth yields for oxygen remain almost constant, because the respiration rates also increase by approximately the same proportion. With high-level expression, the growth rates decrease with further Pps or Pck expression, while the respiration rates remain at the plateau.

Since Pps catalyzes the formation of PEP, it can be concluded that growth on pyruvate with low-level expression is at least indirectly limited by PEP supply, or more accurately, by the Pps activity. It is unlikely that the growth rate on pyruvate is limited by the rate of energy production. On the other hand, because the flux mediated by Pck can lead to either monomer synthesis or the tricarboxylic acid cycle for energy production, we cannot conclude that monomer supply is the rate-limiting flux in cells growing on succinate. Without Pck, however, malic enzyme supports insufficient flux to pyruvate to fuel the tricarboxylic acid cycle and to provide PEP for key metabolite synthesis for optimal growth rate. This explains the low growth rates and the insensitivity to the overproduction of Pps in a $p c k$ $\mathrm{pps}^{+}$strain growing on succinate. The growth enhancement by Pps and Pck in minimal medium supplemented with pyruvate and with succinate, respectively, is unexpected, since Lowry et al. (19) found that PEP concentration is already much higher in cells growing on gluconeogenic substrates than in cells growing on glucose. The maximum growth rates attained by optimal levels of Pps or Pck on pyruvate or succinate both exceed the wild-type growth rates in the same medium and are equal to the wild-type growth rate in glycerol-containing minimal medium. This result is consistent with the hypothesis that the formation of glucose 6-phosphate is a bottleneck for growth in glycerol (20). Overproduction of Pps or Pck presumably increases the supply of PEP and triose phosphates, creating a metabolic state similar to that in the cells growing on glycerol.

The simultaneous increase in the growth rate and the respiration rate with low-level expression results in a constant growth yield with respect to oxygen (Fig. $3 \mathrm{~b}$ and $4 \mathrm{~b}$ ). It appears that the constant yield over a wide range of enzyme levels is a characteristic for efficient growth on a particular carbon source. The cells maintain a constant growth stoichiometry and energy efficiency, which may be optimal for growth on the particular carbon source, even if the growth rate is increased by a higher level of key enzymes. This result contrasts with the yield variation reported by Anderson and von Meyenburg for cells growing on different carbon sources (1).
Further increases in Pps or Pck expression beyond the optimal level reduce the growth rate while the respiration rates remain constant. Consequently, the growth yields for oxygen decrease. Growth inhibition by overproduction of enzymes is not uncommon, particularly when inclusion bodies are formed (3). No inclusion bodies were found in cells overproducing Pps or Pck. Moderate overproduction of soluble enzymes beyond the wild-type level typically has no effect on the growth rate (28). Therefore, growth inhibition caused by the overexpression of Pps and Pck above the optimal levels is unexpected. The underlying mechanism for this effect is unknown, although metabolic perturbations such as futile cycling are possible.

In wild-type $E$. coli, pck expression is regulated at least at the transcriptional level. A study with a $p c k-l a c Z$ operon fusion (14) showed that the transcription of $p c k$-lac $Z$ is induced in cells growing on gluconeogenic media and repressed by glucose and is also induced at the onset of stationary phase in LB medium. The regulation of pps is linked to the PEP-dependent phosphotransferase system $(5,6,11)$. The negative regulator of the fru operon, fruR, has been shown to be required for $p p s$ transcription. pps-lacZ operon fusions showed that the $p p s$ gene in Salmonella typhimurium is under catabolite repression, but the addition of cyclic AMP in the presence of glucose does not relieve this repression (27). Apparently, these regulations have not led to the optimal levels of Pck and Pps for maximal unrestricted growth on succinate and pyruvate.

To our knowledge, Pps and Pck are the only examples of suboptimally produced enzymes in central metabolism, other than transport proteins such as LacY in E. coli (10) and the dicarboxylic acid transport protein ( $d c t$ gene product) in Bradyrhizobium japonicum (2). Other studies have shown that the wild-type levels of $\beta$-galactosidase, GltA, and enzyme II ${ }^{\mathrm{Cilc}}$ of the phosphotransferase system are optimal for $E$. coli growth on lactose (8), acetate (28), and on glucose (25), respectively. Although moderately high levels of Pck and Pps improve growth rate on succinate and pyruvate, they may generate futile cycles during a shift to glucose media. In fact, we have shown that overexpression of Pps under glycolytic conditions generates phenotypes consistent with the futile cycling hypothesis (24). Perhaps because succinate and pyruvate are not common substrates for $E$. coli in its natural habitat, the cell may be optimized for fast shift-up for growth on glucose, rather than optimal growth on the poor substrates.

\section{ACKNOWLEDGMENTS}

This work was supported by the National Science Foundation (grant BCS-9257351 to J.C.L.), Texas Advanced Technology Program (grant 9999(03156 to J.C.L. and R.F.Y.), and the Welch Foundation (grant A1251 to J.C.L.).

\section{REFERENCES}

1. Anderson, K. B., and K. von Meyenburg. 1980. Are growth rates of Escherichia coli in batch cultures limited by respiration? J. Bacteriol. 144:114-123.

2. Birkenhead, K., S. S. Manian, and F. O'Gara. 1988. Dicarboxylic acid transport in Bradyrhizobium japonicum: use of Rhizobium meliloti dct gene(s) to enhance nitrogen fixation. J. Bacteriol. 170:184-189.

3. Bogosian, G., B. N. Violand, P. E. Jung, and J. F. Kane. 1990. Effect of protein overexpression on mistranslation in Escherichia coli, p. 546-558. In W. E. Hill, P. B. Moore, A. Dahlberg, D. Schlessinger, R. A. Garrett, and J. R. Warner (ed.), The ribosome. American Society for Microbiology, Washington. D.C.

4. Chen, W. J., L. Gross, K. E. Joho, and W. T. McAllister. 1992. A modified kanamycin-resistance cassette to facilitate two-codon insertion mutagenesis. Gene 111:143-144.

5. Chin, A. M., D. A. Feldheim, and M. H. Saier, Jr. 1989. Altered 
transcriptional patterns affecting several metabolic pathways in strains of Salmonella typhimurium which overexpress the fructose regulon. J. Bacteriol. 171:2424-2434.

6. Chin, A. M., B. U. Feucht, and M. H. Saier, Jr. 1987. Evidence for the regulation of gluconeogenesis by the fructose phosphotransferase system in Salmonella typhimurium. J. Bacteriol. 169:897899

7. Cooper, R. A., and H. L. Kornberg. 1967. The direct synthesis of phosphoenolpyruvate from pyruvate in Escherichia coli. Proc. R. Soc. London Ser. B. 168:263-280.

8. Dean, A. M., D. E. Dykhuizen, and D. L. Hartl. 1986. Fitness as a function of $\beta$-galactosidase activity in Escherichia coli. Genet. Res. 48:1-8.

9. Degnen, G. E., and E. C. Cox. 1974. Conditional mutator gene in Escherichia coli: isolation, mapping, and effector studies. J. Bacteriol. 117:477-487.

10. Dykhuizen, D. E., A. M. Dean, and D. Hartl. 1987. Metabolic fluxes and fitness. Genetics 115:25-31.

11. Geerse, R. H., J. van der Pluijm, and P. W. Postma. 1989. The repressor of the PEP:fructose phosphotransferase system is required for the transcription of the pps gene of Escherichia coli. Mol. Gen. Genet. 218:348-352.

12. Goldie, A. H., and B. D. Sanwal. 1980. Allosteric control by calcium and mechanism of desensitization of phosphoenolpyruvate carboxykinase of Escherichia coli. J. Biol. Chem. 255:13991405.

13. Goldie, A. H., and B. D. Sanwal. 1980. Genetic and physiological characterization of Escherichia coli mutants deficient in phosphoenolpyruvate carboxykinase activity. J. Bacteriol. 141:11151121.

14. Goldie, H. 1984. Regulation of transcription of the Escherichia coli phosphoenolpyruvate carboxykinase locus: studies with $p c k$-lacZ operon fusions. J. Bacteriol. 159:832-836.

15. Groisman, E. A., and M. J. Casadaban. 1986. Mini-Mu bacteriophage with plasmid replicons for in vivo cloning and lac gene fusing. J. Bacteriol. 168:357-364.

16. Hempfling, W. P., and S. E. Mainzer. 1975. Effects of varying the carbon source limiting growth on yield and maintenance characteristics of Escherichia coli in continuous culture. J. Bacteriol. 123:1076-1087.
17. Ingraham, J. L., O. Maaloe, and F. C. Neidhardt. 1983. Growth of the bacterial cell. Sinauer Associates, Inc., Sunderland, Mass.

18. Jensen, K., and S. Pedersen. 1990. Metabolic growth rate control in Escherichia coli may be a consequence of subsaturation of the macromolecular biosynthesis apparatus with substrates and catalytic components. Microbiol. Rev. 54:89-100.

19. Lowry, O. H., J. Carter, J. B. Wood, and L. Glaser. 1971. The effect of carbon and nitrogen sources on the level of metabolic intermediates in Escherichia coli. J. Biol. Chem. 246:6511-6521.

20. Marr, A. G. 1991. Growth rate of Escherichia coli. Microbiol. Rev. 55:316-333.

21. Medina, V., R. Pontarollo, D. Glaeske, H. Tabel, and H. Goldie. 1990. Sequence of the pckA gene of Escherichia coli K-12: relevance to genetic and allosteric regulation and homology of $E$. coli phosphoenolpyruvate carboxykinase with the enzymes from Trypanosoma brucei and Saccharomyces cerevisiae. J. Bacteriol. 172:7151-7156.

22. Miller, J. H. 1972. Experiments in molecular genetics. Cold Spring Harbor Laboratory, Cold Spring Harbor, N.Y.

23. Niersbach, M., F. Krauzaler, R. H. Geerse, P. W. Postma, and H. J. Hirsch. 1992. Cloning and nucleotide sequence of the Escherichia coli $\mathrm{K}-12$ pps A gene, encoding PEP synthase. Mol. Gen. Genet. 231:332-336.

24. Patnaik, R., W. D. Roof, R. F. Young, and J. C. Liao. 1992. Stimulation of glucose catabolism by a potential futile cycle. J. Bacteriol. 174:7527-7532.

25. Ruyter, G. J. G., P. W. Postma, and K. van Dam. 1991. Control of glucose metabolism by enzyme II $^{\text {Glc }}$ of the phosphoenolpyruvatedependent phosphotransferase system in Escherichia coli. J. Bacteriol. 173:6184-6191.

26. Sambrook, J., E. F. Fritsch, and T. Maniatis. 1989. Molecular cloning: a laboratory manual. Cold Spring Harbor Laboratory Press, Cold Spring Harbor, N.Y.

27. Smyer, J. R., and R. M. Jeter. 1989. Characterization of phosphoenolpyruvate synthase mutants in Salmonella typhimurium. Arch. Microbiol. 153:26-32.

28. Walsh, K., and D. E. Koshland, Jr. 1985. Characterization of rate-controlling steps in vivo by use of an adjustable expression vector. Proc. Natl. Acad. Sci. USA 82:3577-3581. 